\title{
Normativa de regulación jurídica para contribuir a la seguridad industrial en la Cooperativa de Telecomunicaciones
}

\author{
Legal regulation regulations to contribute to Industrial Safety in the \\ Telecommunications Cooperative
}

\section{Regulamentações legais para contribuir com a Segurança Industrial na Cooperativa de Telecomunicações}

Artículo recibido en julio 2019 Arbitrado en agosto 2019 Publicado en octubre 2019

\section{Wilbert Emilio Vedia Ortega} wilbert.vedia@hotmail.com

\section{RESUMEN}

ABSTRACT
La seguridad e higiene del trabajo reside en la protección de la vida y la salud del trabajador. Por tal motivo, el objetivo principal de la investigación fue proponer un proyecto de reglamento jurídico para contribuir a la seguridad industrial en la Cooperativa de Telecomunicaciones. La metodología usada fue, de tipo descriptivo con un enfoque cualitativo y cuantitativo, los métodos usados fueron histórico, lógico, y análisis, síntesis. La técnica e instrumentos que se usó para la recolección de datos fueron estudio documental, encuesta (cuestionario) y entrevista (guía). La población estuvo conformada por 70 trabajadores de la empresa y 4 personas del equipo Directivo. Y la muestra, solo por 20 trabajadores y el total del equipo Directivo. Como resultado se obtuvo que el conocimiento sobre Seguridad Industrial es mínimo en los trabajadores de la empresa. Por lo tanto, con la normativa se permitirá cuidar el bienestar de los trabajadores.

Palabras clave: Seguridad Industrial; derecho laboral; higiene y seguridad, derecho comparado

(he protection of the life and health of the worker. For this reason, the main objective of the research was to propose a draft of legal regulations to contribute to industrial safety in the Telecommunications Cooperative. The methodology used was descriptive with a qualitative and quantitative approach, the methods used were historical, logical, and analysis, synthesis. The technique and instruments used for data collection were a documentary study, survey (questionnaire) and interview (guide). The population was made up of 70 company workers and 4 people from the Management team. And the sample, only by 20 workers and the total of the Management team. As a result, it was obtained that the knowledge about Industrial Safety is minimal in the workers of the company. Therefore, the regulations will allow taking care of the well-being of workers.

Key words: Industrial Safety; labor law; hygiene and safety, comparative law

A segurança e higiene ocupacional estão na proteção da vida e da saúde do trabalhador. Por este motivo, o principal objetivo da pesquisa foi propor um projeto de normativo legal que contribua para a segurança industrial na Cooperativa de Telecomunicações. A metodologia utilizada foi descritiva com abordagem qualitativa e quantitativa, os métodos utilizados foram históricos, lógicos e de análise, síntese. A técnica e os instrumentos utilizados para a coleta de dados foram estudo documental, levantamento (questionário) e entrevista (guia). A população era composta por 70 trabalhadores da empresa e 4 pessoas da equipa de Gestão. E a amostra, apenas por 20 trabalhadores e o total da equipe de Gestão. Como resultado, obteve-se que o conhecimento sobre Segurança Industrial é mínimo nos trabalhadores da empresa. Portanto, a regulamentação vai permitir cuidar do bem-estar dos trabalhadores.

Palavras-chave: Segurança Industrial; lei trabalhista; higiene e segurança, direito comparado 


\section{INTRODUCCIÓN}

Desde la prehistoria, el ser humano ha tenido que protegerse de los riesgos y adversidades del medio que habita. Es así, por ejemplo, que comenzó dándole uso al fuego, construyó herramientas (tales como hachas, cuchillos y puntas de flecha, entre otras) e inventó el arco, probablemente el primer dispositivo que acumulaba energía, a base de piedra y madera, dos elementos abundantes en el medio.

Grimaldi y Simons (1991), plantea que, probablemente, el primer antecedente legal de protección y seguridad existente haya sido el Código de Hammurabi, que data del año 2100 a.c. En este se expresa, de forma detallada, la indemnización por pérdidas o daños, por colisión o construcción de equipos, así como la existencia de tribunales, para conciliar demandas al respecto. Desafortunadamente, el Código aplicaba el principio de "ojo por ojo" cuya intención era la de mantener un control mediante la amenaza explícita de un castigo igual a la gravedad de la ofensa.

Así que, la seguridad industrial en Europa, especialmente en Inglaterra (con la Revolución Industrial), vista como el cumplimiento de normas técnicas por parte de los empleados en un sitio de trabajo, se originó, a partir del incremento de accidentes que se presentaban en las fábricas donde, solo se pensaba en el máximo provecho de la actividad humana con horarios hasta de 14 y 15 horas diarias, incluso niños tuvieron que trabajar junto a sus padres, con el fin de generar lo suficiente para sobrevivir en familia, , enfrentando condiciones de inseguridad. Por lo tanto, los trabajadores debían manejar maquinas sin elementos de protección. Eso sin contar que el sistema mecánico no tenía guardas que los protegiera. Engels, en 1844, al descubrir la situación de Manchester (Inglaterra), donde las máquinas aumentaban sin cesar su potencia y velocidad, creando cada vez mayores peligros. En consecuencia, en Inglaterra, se efectúan los primeros intentos formales por proteger la salud de los trabajadores. Así que, en el año 1802 se aprobó la primera ley relativa a la "salud y moral de los aprendices" y en 1844 se aplicó la primera norma que protegía a las mujeres en el trabajo. Así mismo en 1850, se inicia las primeras inspecciones en los trabajos de las minas, reguladas por el gobierno Inglés.

Seguidamente, hacia fines del siglo XIX, la seguridad industrial cobró relevancia, pues los gobiernos de muchos países se preocuparon por desarrollar una legislación al respecto. De esta forma, pronto otros países europeos, especialmente los Escandinavos, Alemania, Italia y Rusia, establecen sistemas de inspección en las fábricas, reglamentos de trabajo y legislaciones sobre indemnizaciones por accidentes ocasionados en el desarrollo del mismo.

Por otra parte en Massachusetts y Detroit (Estados Unidos), a fines de siglo XIX, se inició el desarrollo de la legislación laboral, que luego se extiende a los demás estados. De esta manera, se crean eficientes entidades privadas interesadas en la 
seguridad industrial, que manejan conceptos claros aceptados por patrones, obreros y usuarios, sobre lo que debe ser la prevención de accidentes de los trabajadores, no solo en el espacio laboral, sino en el hogar y en la calle.

Sin embargo, en Bolivia, no es una novedad, que en la rutina diaria, se conozca por los medios de comunicación, de los innumerables incidentes y accidentes, sucedidos en la ciudad, departamento y país. Todo por la poca seguridad ciudadana, por un lado, y la poca importancia que se le da a la seguridad personal (empleadores y empleados), por otro lado, no se tiene cultura respecto a la seguridad y será demasiado difícil implantar las normas y hacer cumplir las leyes, que ya existen al respecto.

La solución es formar nuevos ciudadanos con conocimiento y conciencia de la seguridad, lo cual se consigue en las aulas, a través de profesores y catedráticos. Lo lamentable es que en los programas de colegio, no se toma en cuenta esta materia y lo más lamentable aun, es que en la Universidad, son pocas las carreras que la incluyen. (Camargo, 1999).

Cabe destacar, que la regulación en materia de Seguridad Industrial, marca una total preocupación por la poca importancia que le dan los ejecutivos y empresarios a estas normas de Seguridad Industrial, necesarias para la protección y prevención de la vida, enfermedades y accidentes de los trabajadores, quienes sin estas normas jurídicas están propensos a sufrir daños severos en su integridad.

No obstante, los trabajadores de la Cooperativa de Telecomunicaciones Potosí Ltda., ejercen sus labores en condiciones de inseguridad industrial a falta de una regulación interna jurídica actual y concordante con la legislación vigente sobre la temática Por ello, la investigación tiene como objetivo principal proponer un proyecto de reglamento de regulación jurídica para contribuir a la seguridad industrial en la Cooperativa de Telecomunicaciones Potosí Ltda. De esta manera, se garantizará un avance a nivel legislativo en Bolivia.

\section{Marco teórico}

\section{El derecho de trabajo}

El derecho de trabajo es una de esas ramas jurídicas que encierran mucha complejidad en la resolución de sus problemas, en principio porque da cabida a la aplicación de otras fuentes supletorias para la resolución de casos no contemplados en la ley; porque orienta en cierto sentido la interpretación, cuando exista duda sobre ésta; y orienta a preferir ciertas normas con lo cual hace una exclusión de ciertas 
leyes; porque debido a su constante desarrollo, obliga a las autoridades encargadas a velar por su aplicación, sometiéndose a la necesidad de practicar una integración de leyes dispersas.

A su vez, los procedimientos para la aplicación del derecho de trabajo, resulta conveniente reflexionar sobre lo que se ha llamado Jurisprudencia Técnica, la cual según García (2006) "tiene por objeto la exposición ordenada y coherente de los preceptos jurídicos que se hallan en vigor en una época y en un lugar determinados, y el estudio de los problemas relativos a su interpretación y aplicación" (s/p). Explica García, ob. cit. que, la jurisprudencia técnica en su aplicación procede de manera dogmática, no examina la justificación de las disposiciones que componen el derecho positivo. Para la jurisprudencia técnica el orden positivo es un conjunto de reglas cuyo valor no se discute.

\section{Seguridad industrial}

La seguridad industrial es un área multidisciplinaria que se encarga de minimizar los riesgos en la industria. Parte del supuesto de que toda actividad industrial tiene peligros inherentes que necesitan de una correcta gestión. (Camargo, 1999).

Es el conjunto de acciones que permiten localizar y evaluar los riesgos, y establecer las medidas para prevenir los accidentes de trabajo.

"El trabajar sin seguridad ocasiona incidentes que en el peor de los casos se convierten en accidentes" (Valenzuela, 2010).

A modo de síntesis se puede manifestar que, aplicada a los centros de trabajo, la seguridad y la higiene tiene como objetivo salvaguardar la vida y preservar la salud y la integridad física de los trabajadores por medio del dictado de normas encaminadas tanto a que les proporcionen las condiciones para el trabajo, como a capacitarlos y adiestrarlos para que se eviten, dentro de lo posible, las enfermedades y los accidentes laborales.

De ahí que, la seguridad e higiene industrial, es el conjunto de conocimientos científicos y tecnológicos destinados a localizar, evaluar, controlar y prevenir las causas de los riesgos en el trabajo a que están expuestos los trabajadores en el ejercicio o con el motivo de su actividad laboral. Por tanto, es importante establecer que la seguridad y la higiene son instrumentos de prevención de los riesgos y deben considerarse sinónimos por poseer la misma naturaleza y finalidad. (Mariaca, 2009).

\section{Campo de acción de la higiene y la seguridad industrial}

La Higiene y Seguridad Industrial, trata sobre los procedimientos para identificar, evaluar y controlar los agentes nocivos y factores de riesgo, presentes en el medio ambiente laboral, bajo ciertas circunstancias, son capaces de alterar la integridad 
física y/o psíquica del ser humano; ya que estos procedimientos son reglamentados legalmente, considerando que la ley protege al trabajador desde su hogar para trasladarse a su centro de trabajo, su acción recae en la vida cotidiana del trabajador, pues también existen riesgos tanto en el hogar como en todos los servicios públicos. Por consiguiente, hace uso de: la medicina del trabajo, la psicología laboral, la ingeniería, la administración de trabajo, entre otros.

De esta manera, se puede decir, que el campo de acción comprende entre otros ámbitos, los siguientes: las condiciones materiales del ejercicio del trabajo, esfuerzo, fatiga, temperatura, ventilación, presencia de agentes contaminantes, el interés de la propia tarea, monotonía, el carácter competitivo de la tarea, las estimulaciones, la tensión y las cargas mentales, las oportunidades de aprender algo nuevo y adquirir una calificación mayor, ser promocionado, duración de la jornada, grado de exposición a los agentes contaminantes, el rotar turnos, grado de flexibilidad y carácter de los supervisores y todo lo que se pueda concebir que corresponda al ambiente laboral. (Mariaca, 2009)

\section{Ventajas de la seguridad e higiene industrial}

Implementar y llevar a efecto programas de Seguridad e Higiene para lograr un ambiente seguro en el área de trabajo y que los trabajadores trabajen seguramente y con tranquilidad, es parte integral de la responsabilidad total de todos, ya que haciendo conciencia, a todos acarrearía beneficios.

La reducción de riesgos laborales automáticamente disminuirá los costos de operación y aumentaría las ganancias (pues en la aplicación efectiva de los programas, está el objetivo primordial, el de obtener ganancias). Controlar las observaciones y las causas de pérdidas de tiempo relacionadas con la interrupción del trabajo efectivo; aumentar el tiempo disponible para producir, evitando la repetición del accidente, y reducir el costo de las lesiones, incendios, daños a la propiedad, y crear un mejor ambiente laboral. (Mariaca, 2009).

\section{Repercusiones negativas de la falta de seguridad e higiene}

De los efectos negativos que el trabajo puede tener para la salud del trabajador, cabe mencionar, los accidentes dado a que son los indicadores inmediatos y más evidentes de las malas condiciones del lugar de trabajo, y dada su gravedad, la lucha contra ellos es el primer paso de toda actividad preventiva; los altos costos que genera, no son las únicas consecuencias negativas; el Seguro Social, no resucita a los muertos; no puede devolver los órganos perdidos, que cause una incapacidad laboral permanente: Además los sufrimientos físicos y morales que padece el trabajador y su familia, los riesgos reducen temporalmente o definitivamente la posibilidad de 
trabajar, siendo un freno para el desarrollo personal del individuo como ser transformador, ya que le priva total o parcialmente de poderse realizar como miembro activo de la sociedad. Las pérdidas son generalmente los costos directos y son fácilmente cuantificables, ya que involucran el costo de los equipos, edificios y materiales; además existen los costos, tales como: pago de indemnización, pérdida de la producción, del mercado, entrenar a personal de reemplazo, etc. (Mariaca, 2009).

\section{Legislación boliviana en materia de seguridad industrial, salud y seguridad en el trabajo}

Para el desarrollo de la investigación se tomaron como base varios estamentos presentes en la Ley General de Higiene, Seguridad Ocupacional y Bienestar, Ley General del Trabajo y la Constitución Política del Estado.

Ley General de Higiene, Seguridad Ocupacional y Bienestar aprobada en 1979 propone:

- Garantizar las condiciones adecuadas de salud, higiene, seguridad y bienestar en el trabajo.

- Lograr un ambiente de trabajo desprovisto de riesgos para la salud psicofísica de los trabajadores.

- Proteger a las personas y al medio ambiente, contra los riesgos que, directa o indirectamente, afectan a la salud, la seguridad y el equilibrio ecológico.

En el tiempo esta ley, una de las más avanzadas para su época, no ha logrado ser aplicada a cabalidad, razón por la cual, la situación de la seguridad y salud en el trabajo, es precaria.

La Ley General del Trabajo en el título V, "De la seguridad e higiene en el trabajo" contempla las disposiciones generales que deben regular las condiciones generales del trabajo con el objetivo de "proteger la vida, salud y moralidad de los trabajadores".

Finalmente, la Constitución Política del Estado, como norma fundamental del ordenamiento jurídico, consagra en el artículo 15 , los derechos fundamentales de las personas y en el Capítulo Cuarto, Sección II del "Régimen Social", todas las garantías y obligaciones para preservar el trabajo y el capital humano. (Mariaca, 2013). 


\section{Derecho Comparado}

\section{Legislación de Costa Rica. Accidentes Laborales}

Según la legislación (Código Laboral 1995) de Costa Rica se señala que:

Si durante la jornada de trabajo se sufre algún tipo de accidente, recuerda que se tiene derecho a las siguientes prestaciones: asistencia médico - quirúrgica, hospitalaria, farmacéutica y de rehabilitación, prótesis y aparatos médicos que necesites para corregir deficiencias funcionales, prestaciones en dinero que, como indemnización por incapacidad temporal, permanente o por la muerte, están establecidas por ley (y que puedes buscar en el código de trabajo), gastos de traslado, gastos de hospedaje y alimentación, cuando, con motivo del suministro de las prestaciones médico - sanitarias o de rehabilitación, tengas que trasladarte a un lugar diferente de tu residencia habitual o lugar de trabajo, readaptación, reubicación y rehabilitación laboral, para recuperar la normalidad en tu puesto de trabajo.

Además, si ocurre un accidente de trabajo y no se está asegurado, se tendrá derecho a ser igualmente atendido si se acude al Instituto Nacional de Seguros, o a cualquier hospital, clínica o centro de salud, público o privado.

Seguidamente, durante la incapacidad temporal, se tendrá un derecho a un subsidio igual al $60 \%$ del salario diario durante los primeros cuarenta y cinco días de incapacidad. Transcurrido ese plazo, el subsidio será equivalente al $100 \%$ del salario diario, si percibiere una remuneración diaria igual o inferior a cien colones. Por el contrario, si el salario es superior a cien colones por día, sobre el exceso se pagará un subsidio igual al $67 \%$.

\section{Legislación de Chile}

La legislación Chilena, en cuanto a Accidentes de Trabajo y Enfermedades Profesionales, se puede dividir en cuatro periodos:

- Periodo de la legislación Indiana.

- Periodo de la legislación Aislada.

- Periodo de las primeras Leyes Específicas.

- Periodo actual Ley 16.744.

\section{Legislación de Venezuela}

En la nueva Ley del trabajo se desarrolla el Capítulo VI sobre la Higiene y Seguridad en el trabajo, desde el artículo 236 hasta 246.

Con apoyo a la legislación comparada, se entiende que la seguridad industrial es una obligación que la ley impone a patrones y a trabajadores la observancia y 
cumplimiento de las descripciones. Por lo que, el, patrón estará obligado a observar, cada una de las instrucciones o reglas que se dan a establecer para el manejo o conocimiento de un arte o facultad, sobre la seguridad en las instalaciones de su empresa, y a adoptar las medidas adecuadas para prevenir accidentes en el empleo y manipulación de objetos, instrumentos y materiales de trabajo, a fin de garantizar la salud y la vida de los trabajadores, y la responsabilidad social del empresario. En tal sentido, las leyes contendrán al efecto, las sanciones procedentes en cada caso. Por lo cual patrón y trabajadores, se deben organizar dentro de planes determinados, para hacer funcionar dentro de los procedimientos pautados, la seguridad de los trabajadores y de la actividad laboral.

\section{MÉTODO}

Fue de tipo descriptiva, bajo un enfoque mixto, cualitativo y cuantitativo, los métodos usados fueron histórico-lógico, con la finalidad de estudiar el problema en su desarrollo histórico analizando la seguridad industrial en el ámbito de la legislación jurídica y análisis - síntesis, con la finalidad de sistematizar la información sobre el tema y de los diferentes criterios jurídicos para establecer la normativa de regulación de la seguridad industrial.

Las técnicas e instrumentos usados durante la investigación fueron Estudio documental. Con la finalidad de acopiar información acerca de la seguridad industrial y también para el acopio de información sobre normativas de regulación jurídica en el ámbito de la seguridad industrial. Encuesta a trabajadores en donde se realizó un estudio diagnóstico sobre la problemática a investigar. El instrumento utilizado fue el cuestionario, consistió en la estructuración de preguntas o ítems acerca de la seguridad industrial en la empresa. Este instrumento permitió la recolección de datos provenientes de fuentes primarias, en nuestro caso, de las personas seleccionadas. Entrevista al equipo directivo de la cooperativa. El instrumento utilizado fue la guía de entrevista, se aplicó al equipo directivo de la empresa con el propósito de contrastar la opinión de los trabajadores sobre la seguridad industrial, ya que, los integrantes del equipo directivo son profesionales con formación académica reconocida, por lo que sus opiniones evidentemente son más técnicas y de mayor fidelidad.

Por otro lado, la población utilizada para el desarrollo de la investigación fueron los 70 trabajadores de la empresa y 4 personas del equipo Directivo de la empresa. La muestra que se utilizó fueron 20 trabajadores de la empresa y las 4 personas del equipo Directivo de la empresa. 


\section{RESULTADO Y DISCUSIÓN}

La construcción del cuestionario aplicado de los trabajadores de la empresa ha seguido un largo recorrido. La elaboración inicial del instrumento, empezó por el estudio de fundamentación teórica en el marco de los estudios realizados por el investigador sobre "reflexiones jurídicas sobre la seguridad industrial en las cooperativas de servicios".

Análisis de resultados según los ítems del cuestionario aplicado a los trabajadores.

\section{Conoce la normativa sobre seguridad industrial}

Tabla 1. Normativa sobre Seguridad Industrial

\begin{tabular}{ccc}
\hline ALTERNATIVAS & FRECUENCIA & $\%$ \\
\hline $\mathrm{Si}$ & 14 & 20 \\
No & 16 & 80 \\
TOTAL & $\mathbf{2 0}$ & $\mathbf{1 0 0}$
\end{tabular}

A partir de la tabla 1 se muestra que, el $80 \%$ (16 trabajadores) contestaron a la pregunta con la respuesta NO. Solo el 20\% (4 trabajadores) de los trabajadores expresa la respuesta SI. Estos datos reflejan un desconocimiento mayoritario sobre la normativa existente sobre la Seguridad Industrial en la Empresa. Esta situación posibilita contar con un espacio fértil para la incorporación de una normativa reguladora de la seguridad industrial en la empresa.

\section{Conocimiento de la seguridad industrial en la empresa}

Se muestra en la tabla 2, que el $98 \%$ de los trabajadores coincidieron en señalar que es importante conocer sobre la seguridad industrial en la empresa y solo el $2 \%$ consideró que no es vital conocer sobre la seguridad industrial.

Tabla 2. Cocimiento sobre la Seguridad Industrial en la empresa.

\begin{tabular}{ccc}
\hline ALTERNATIVAS & FRECUENCIA & $\%$ \\
\hline $\mathrm{Si}$ & 19 & 98 \\
$\mathrm{No}$ & 1 & 2 \\
TOTAL & $\mathbf{2 0}$ & $\mathbf{1 0 0}$ \\
\hline
\end{tabular}


Estos resultados obtenidos, permitieron concluir la gran necesidad que tienen los trabajadores de conocer sobre la seguridad industrial, lo que implicó la necesidad de establecer espacios en la empresa orientados a la implementación urgente de una normativa jurídica que regule la seguridad industrial en la empresa.

\section{Conoce el documento normativo sobre seguridad industrial de la empresa}

En la tabla 3, se muestra que el $75 \%$ de los trabajadores manifestaron que no conocen el documento normativo sobre la seguridad industrial en la empresa. En cambio un $25 \%$ manifestaron que conocen sobre la normativa. Lo que significa que un alto porcentaje de los trabajadores se encuentran en riesgo laboral por su desconocimiento. Esto implica la necesidad de que la empresa a través del equipo directivo establezca mecanismos que permitan que la mayoría de los trabajadores conozcan el documento normativo. Así mismo, las distribuciones porcentuales, muestran el carácter incipiente respecto al conocimiento de la normativa.

Tabla 3. Documento normativo sobre seguridad industrial de la empresa

\begin{tabular}{ccc}
\hline ALTERNATIVAS & FRECUENCIA & \% \\
\hline $\mathrm{Si}$ & 5 & 25 \\
$\mathrm{No}$ & 15 & 75 \\
TOTAL & $\mathbf{2 0}$ & $\mathbf{1 0 0}$ \\
\hline
\end{tabular}

Puesto de trabajo, cartillas, guías, manuales o reglamentos sobre Seguridad Industrial

En la tabla 4 se muestra que el $85 \%$ de los trabajadores respondieron NO y el $15 \%$ respondieron $\mathrm{SI}$, en relación a la existencia de manuales o reglamentos acerca de la seguridad industrial

Tabla 4. Presencia de manuales o reglamentos sobre seguridad industrial en el trabajo.

\begin{tabular}{ccc}
\hline ALTERNATIVAS & FRECUENCIA & \% \\
\hline Si & 3 & 25 \\
No & 17 & 85 \\
TOTAL & $\mathbf{2 0}$ & $\mathbf{1 0 0}$ \\
\hline
\end{tabular}

Los resultados revelaron que en la empresa existen insuficiencias referidas a la comunicación explícita de los documentos normativos relativos a la seguridad 
industrial, lo que significa el aumento de los riesgos laborales en los trabajadores por desconocimiento. Este aspecto implicó que la empresa debe realizar los esfuerzos necesarios para establecer los medios para que los trabajadores tengan acceso permanente a la información jurídica sobre la seguridad industrial.

\section{Información sobre las medidas de seguridad que se debe tener en el puesto de trabajo}

En la tabla 5 se muestra que el $30 \%$ de los trabajadores respondieron afirmativamente en cambio el $70 \%$ de los trabajadores encuestados respondieron negativamente, acerca de la dimensión sobre las medidas de seguridad en los puestos de trabajo.

Tabla 5. Medidas de seguridad

\begin{tabular}{ccc}
\hline ALTERNATIVAS & FRECUENCIA & $\%$ \\
\hline $\mathrm{Si}$ & 6 & 30 \\
$\mathrm{No}$ & 14 & 70 \\
TOTAL & $\mathbf{2 0}$ & $\mathbf{1 0 0}$ \\
\hline
\end{tabular}

En este sentido, los resultados obtenidos, revelaron que en un porcentaje mínimo reconocen que la empresa ha logrado informar a los trabajadores de las medidas de seguridad que deben tener en su puesto de trabajo. En cambio un gran porcentaje expresaron que no han recibido de parte de la empresa información respecto a las medidas de seguridad. Lo que induce a expresar que la empresa debe tomar acciones que permitan llegar a todos los trabajadores con la información sobre las medidas de seguridad con fines de prevención de riesgos y por ende garantizar la salud de los trabajadores.

\section{Entrevista al equipo directivo de la empresa}

En las entrevistas participaron las cuatro personas clave identificadas del equipo directivo de la Cooperativa (Gerente General, Asesor Legal, Gerente Técnico, Director Administrativo financiero). Bajo la dimensión establecida en el guion de entrevista respecto a la seguridad industrial en la Cooperativa se obtuvo la siguiente distribución de opiniones sistematizadas.

\section{Conocimiento de la seguridad industrial}

Los entrevistados en el orden del $95 \%$ expresaron que tienen conocimientos generales y básicos sobre la legislación jurídica en materia de la seguridad Industrial, sin embargo, no conocen la normativa existente en la Cooperativa sobre la seguridad Industrial. 


\section{Aplicación de la normativa sobre la seguridad industrial}

El $85 \%$ de los entrevistados, manifestaron que no tienen un documento claro sobre la normativa de la Seguridad Industrial en la Cooperativa, razón por la cual, no se aplica en la cooperativa.

\section{Socialización sobre seguridad industrial en la empresa}

El equipo directivo, en su conjunto coincidieron en señalar que no se ha efectivizado una socialización sobre la Seguridad Industrial en la empresa, porque no cuenta con el Reglamento específico. Solo reconocen que ocasionalmente se invita a algún experto en seguridad industrial para que realice información sobre normativas jurídicas existentes.

A partir de estos resultados obtenidos de las entrevistas realizadas al equipo directivo de la Cooperativa, se pudo evidenciar que la Cooperativa no cuenta con un Reglamento General sobre Seguridad Industrial, que garantice la salud integral de los trabajadores y promueva la prevención de los riesgos en la Cooperativa.

Hecho el análisis de los resultados obtenidos mediante la aplicación de los instrumentos, se contrastó la información obtenida y se sistematizaron un conjunto de condiciones que permitieron realizar la caracterización del estado actual en el que se encuentra la Seguridad Industrial en la Cooperativa.

- El conocimiento acerca de la seguridad industrial en la Cooperativa se encontró en un estado incipiente en los trabajadores.

- Los criterios expresados por los trabajadores y corroborados por los directivos de la Cooperativa confirmaron la inexistencia de una normativa jurídica reguladora de la seguridad industrial en la Cooperativa.

Por tanto, se infiere categóricamente la existencia de una contradicción entre el estado ideal y la situación actual sobre la seguridad industrial en la Cooperativa de Telecomunicaciones Potosí Ltda. Lo que implicó la demanda urgente de una propuesta que permita transformar esta realidad y aproximarla a un estado deseado.

\section{Propuesta de Normativa de regulación jurídica en la Cooperativa de Telecomunicaciones Potosí Ltda}

La propuesta, en su esencia revelará los elementos jurídicos que debe tener una normativa jurídica de seguridad industrial en la Cooperativa. De esta manera, el objetivo de la propuesta será dotar a la Cooperativa de una normativa jurídica en materia de Seguridad Industrial. El propósito de la propuesta, será establecer una 
normativa de regulación jurídica en Seguridad Industrial en la Cooperativa. Y el desarrollo estará orientado a incorporar todos los aspectos y/o elementos jurídicos sobre la seguridad industrial en el documento denominado "Normativa de regulación jurídica de la Seguridad Industrial en la Cooperativa de Telecomunicaciones Potosí Ltda."

\section{Fundamentación jurídica}

Para la creación de la propuesta se tomó en cuenta los fundamentos jurídicos de la Constitución Política del Estado, a partir de la cual se derivan cualquiera de las normativas o reglamentos en actual vigencia en el Estado Plurinacional de Bolivia.

Seguidamente, la Normativa de Seguridad Industrial para la Cooperativa de Telecomunicaciones Potosí Ltda., está estructurada de la siguiente manera:

Título I Generalidades.

- Objetivos

- Alcance de la norma

- Prevención de accidentes

- Términos y definiciones

- Liderazgo, compromiso y políticas de seguridad

- Liderazgo

- Compromiso

- Políticas de seguridad industrial

- Reglas de oro de la seguridad

Título II. Obligaciones y derechos

- Obligaciones de la empresa

- Derechos y obligaciones de los trabajadores

- Comité mixto de seguridad industrial

- Empresas contratistas

Título III. Reconocimientos, infracciones y sanciones

- Reconocimientos

- Infracciones

- Sanciones

- Responsabilidades del encargado

- Responsabilidades de los empleados 
Título IV Sistema de distribución

- Generalidades

- Procedimientos y autorización

- Requisitos específicos para el sistema de distribución

Titulo V. Estándares de seguridad en las operaciones

- Manejo de equipo pesado, carretas de cables de alta capacidad y herramientas.

Título VI. Estándares de peligros existentes y riesgos evaluados

Título VII. Equipo de protección personal

Criterios generales para la selección de los equipos de protección

- Protección de la cabeza

- Protección facial

- Protección visual

- Protección de las extremidades inferiores (pies)

- Protección de las manos

- Protección auditiva

- Protección respiratoria

- Uniforme de trabajo y/o ropa de trabajo

- Cinturones de seguridad y arnés

- Sogas o correas

- Equipo revelador o detector de tensión

- Pértigas

- equipo de puesta a tierra

- Herramientas aisladas

Título VIII. Preparación y respuesta ante emergencias

- Prevención y control de incendios

- Control de incendios en ambientes de centrales y subcentrales

- Brigadas de emergencia

- Programación de simulacros de lucha contra incendios

- Manipuleo de equipos de planta en caso de incendios 
Asistencia médica, primeros auxilios y examen médico

- Capacitación en primeros auxilios

- Traslado de accidentados

- Botiquines de primeros auxilios

- Sobre los vehículos

Título IX Gestión de accidentes

- Procedimiento para el reporte de incidentes

- Procedimiento de investigación de incidentes

- Estadísticas de incidentes de trabajo

Título X. Capacitación y entrenamiento

- Capacitación y entrenamiento

- Enfoque de la capacitación

\section{CONCLUSIONES}

Partiendo del objetivo central de la investigación, se ha podido establecer que es una necesidad primordial la creación de la normativa de regulación jurídica en materia de Seguridad Industrial.

Para finalizar, la normativa ofrece una perspectiva real de implementación en la Cooperativa condecente a mejorar la situación actual diagnosticada. Principalmente, la propuesta va orientada a establecer una regulación jurídica condecente a velar la seguridad integral de los trabajadores, cuyo impacto inmediato será la de cuidar el bienestar de los trabajadores.

\section{REFERENCIAS}

Camargo, D. (1999). Comentarios al procedimiento laboral. Bolivia: Editores Universitarias

Constitución Política del Estado Plurinacional de Bolivia. (2009)

García, A. (2006). Tratado elemental de Seguridad en el trabajo. Argentina. Ediciones KAPELUZ

Grimaldi, A., y Simons, C. (1991). Instituciones del derecho individual del trabajo. Guatemala. Ediciones FENIX

Ley General de Higiene, Seguridad Ocupacional y Bienestar (aprobada por Decreto Ley n016998, del 2 de agosto de 1979). Bolivia
Ley General del Trabajo de la República Plurinacional de Bolivia (1992)

Ley 16.744 (2011). Ley sobre accidentes del trabajo y enfermedades profesionales. República de Chile.

Mariaca, G. (2009). Los principios del derecho de trabajo. España: Editorial Montecorro, S.A.

Sistema Costarricense de Información Jurídica. (1995). El Código de Trabajo de la República de Costa Rica

Valenzuela, H. (2010). Breve historia del derecho laboral internacional. México: Editorial UNIVERSAL 